\title{
Yukawa coupling and anomalous magnetic moment of the muon: an update for the $\mathrm{LHC}$ era
}

\author{
Andreas Crivellin ${ }^{1,2}$, Jennifer Girrbach ${ }^{1}$, and Ulrich Nierste ${ }^{1}$ \\ ${ }^{1}$ Institut für Theoretische Teilchenphysik \\ Karlsruhe Institute of Technology, Universität Karlsruhe, \\ D-76128 Karlsruhe, Germany. \\ ${ }^{2}$ Albert Einstein Center for Fundamental Physics, \\ Institute for Theoretical Physics, \\ University of Bern, CH-3012 Bern, Switzerland.
}

(Dated: October 2010)

\begin{abstract}
We study the interplay between a soft muon Yukawa coupling generated radiatively with the trilinear $A$-terms of the minimal supersymmetric standard model (MSSM) and the anomalous magnetic moment of the muon. In the absence of a tree-level muon Yukawa coupling the lightest smuon mass is predicted to be in the range between $600 \mathrm{GeV}$ and $2200 \mathrm{GeV}$ at $2 \sigma$, if the bino mass $M_{1}$ is below $1 \mathrm{TeV}$. Therefore, a detection of a smuon (in conjunction with a sub-TeV bino) at the LHC would directly imply a nonzero muon Yukawa coupling in the MSSM superpotential. Inclusion of slepton flavor mixing could in principle lower the mass of one smuonlike slepton below $600 \mathrm{GeV}$. However, the experimental bounds on radiative lepton decays instead strengthen the lower mass bound, with larger effects for smaller $M_{1}$, We also extend the analysis to the electron case and find that a light selectron close to the current experimental search limit may prove the MSSM electron Yukawa coupling to be nonzero.
\end{abstract}

PACS numbers: 14.80.Ly,12.60.Jv,13.40.Em

\section{INTRODUCTION}

In the standard model (SM) the measured fermion masses determine the values of the Yukawa couplings. Beyond the standard model, however, the Yukawa sector is terra incognita: In the minimal supersymmetric standard model (MSSM), which we consider in this paper, the relations between the masses $m_{f}$ and the Yukawa couplings $y_{f}$ depend on the ratio of the two vacuum expectation values $\tan \beta=v_{u} / v_{d}$ and further receive important radiative contributions from the soft supersymmetrybreaking sector. In the decoupling limit $M_{\text {SUSY }} \gg$ $M_{A^{0}}, M_{H^{+}}, v_{u}$ the effective loop-induced Yukawa coupling arise in an intuitive way from one Higgs coupling to sfermions, which involve either the trilinear terms $A_{i j}^{f}$ ( $f=u, d, \ell$ and $i, j=1,2,3$ label the generation) or the Higgsino mass parameter $\mu$ accompanied by $y_{f}$, by integrating out the heavy SUSY particles [1, 2, 3, 4, 4, 5, 6, , 7, 8]. However, if no hierarchy between the sparticle mass scale $M_{\text {SUSY }}$, the Higgs masses, and the vevs is present, one has to calculate the self-energy diagrams using exact diagonalization (which correctly accounts for all powers of $A$-terms and $\left.\mu Y^{f}\right)$. This then leads to a finite renormalization of the Yukawa couplings and mixing matrices of quarks [9, 10, 11] and leptons [12]. With the appropriate all-order resummations the result for the Yukawa coupling reads:

$$
y_{f_{i}}=\frac{m_{f_{i}}-\Sigma_{i i, A}^{f L R}}{v_{u, d}\left(1+\Sigma_{i i, \mu}^{f L R} /\left(y_{f_{i}} v_{u, d}\right)\right)} .
$$

In Eq. (1) we have decomposed the self-energy $\Sigma_{i i}^{f L R}$ as $\sum_{i i, A}^{f L R}+\Sigma_{i i, \mu}^{f L R}$ as in [11]. $\sum_{i i, \mu}^{f L R}$ is proportional to $\mu y_{f_{i}}$ and $\Sigma_{i i, A}^{f L R}$ is the remaining part of the self-energy, in which the chirality-flip does not stem from $y_{f_{i}}$, but e.g. from $A_{i i}^{f}$. Equation (11) is only correct for negligible flavor-mixing. Furthermore, since the size of $\Sigma_{i i, A}^{f L R}$ can be of the order of the light fermion masses, we do not even know if the light fermions possess a tree-level Yukawa coupling at all, because it might be possible that their masses are entirely generated by the radiative contribution 11, 11, 13, 14, 15, 16, 17, 18, 19, 20, 21, 22, 23. For a vanishing hard Yukawa coupling $y_{f}$ in the superpotential one has $\Sigma_{i i, \mu}^{f L R}=0$ at the one-loop level. However, the same trilinear term $A_{i i}^{f}$ needed to generate the soft contribution stemming from $\Sigma_{i i, A}^{f L R}$ in eq. (1) also enters the anomalous magnetic moment of the corresponding fermion [20]. The anomalous magnetic moment of the muon, $a_{\mu}=\frac{1}{2}(g-2)_{\mu}$, is of special importance since its precisely measured value deviates from the SM prediction by more than $3 \sigma[24,25,26,27,28$, 29, 30]. An improved $(g-2)_{\mu}$ experiment could decrease the experimental error by a factor of 4 [31]. The interplay between $a_{\mu}$ and a radiatively generated muon mass was already studied in [20]: If $A_{22}^{\ell}$ is adjusted to reproduce $m_{\mu}$, one tends to overshoot the desired new-physics contribution to $a_{\mu}$.

In the next section we will update the 1999 analysis of 20] using present-day inputs from experiment and theory. We extend this study by including the effects of slepton mixing, which can lead to additional contributions proportional to $y_{\tau}$. With sufficiently heavy bino and smuon masses one can generate $m_{\mu}$ from the nondecoupling soft loop contribution $\Sigma_{22, A}^{f L R}$ and simultaneously satisfy the $a_{\mu}$ constraint which disappears in the decou- 
pling limit. Therefore, the discoveries of these particles at the LHC will eventually permit to rule out an entirely soft muon mass and instead establish a nonzero $y_{\mu}$ in the superpotential. The aim of this paper is to quantify this statement. In addition, we investigate the electron case.

\section{CORRELATION BETWEEN $a_{\mu}$ AND $y_{\mu}$}

The magnetic dipole moment interaction relevant for $a_{\mu}$ is given by

$$
\frac{i e}{2 m_{\mu}} F\left(q^{2}\right) \bar{u}\left(p_{f}\right) \sigma_{\mu \nu} q^{\mu} \epsilon^{\nu} u\left(p_{i}\right)
$$

where $q=p_{f}-p_{i}$ is the momentum and $\epsilon$ is the polarization vector of the external photon. The anomalous magnetic dipole moment of the muon is then given as

$$
a_{\mu}=F\left(q^{2}=0\right) \text {. }
$$

Experimentally, $a_{\mu}$ differs from its SM prediction by $3.6 \sigma$ 30]:

$$
a_{\mu}^{\exp }-a_{\mu}^{\mathrm{SM}}=(28.7 \pm 8.0) \times 10^{-10} .
$$

In unbroken supersymmetric theories the gryomagnetic ratio for all fermions is exactly 2 32]. Therefore, the anomalous magnetic moment of the muon directly probes SUSY breaking. A pleasant feature of supersymmetry, which distinguishes it from many alternative theoretical frameworks, is that it can naturally explain the observed deviation from the SM value [33, 34, 35, 36, 37, 38, 39, 40, 41]. The usual approach is to choose a suitable (large) value of the term $\left|y_{\mu}\right| \mu v_{u} \approx m_{\mu} \mu \tan \beta$ [59]. In order to achieve the right value for the anomalous magnetic moment, the higgsino mass parameter $\mu$ must be positive and large values for $\tan \beta \gtrsim 10$, the ratio of the two vacuum expectation values, are favored. While large$\tan \beta$ scenarios are also motivated by the GUT relation $\left|y_{t}\right|=\left|y_{b}\right|$, problems in processes like $b \rightarrow s \gamma, B_{d, s} \rightarrow$ $\mu \bar{\mu}$ and $B \rightarrow(D) \tau \nu$ can occur, due to the parametric enhancement by $\tan \beta$ [7, 8, 42, 43]. In mSUGRA and the constrained MSSM, $B_{d, s} \rightarrow \mu \bar{\mu}$ and the anomalous magnetic moment of the muon are correlated, limiting the possible size of $a_{\mu}^{\mathrm{SUSY}}$ [39]. Therefore, if $\tan \beta$ is large, the nonstandard Higgses have to be heavy in the CMSSM [44].

However, there exists also a second, less studied way in the MSSM to account for the anomalous magnetic moment of the muon: The entry in the slepton mass matrix involving the trilinear SUSY-breaking terms, $v_{d} A_{22}^{\ell}+$ $v_{u} A_{22}^{\ell \prime}$, can also reproduce the desired effect without influencing quark decays or the Higgs potential. This possibility is realized in models with radiative generation of fermion masses [11, 15, 20, 21, 22]. In these models the trilinear terms are chosen in such a way that they generate the light fermion masses of the first and second generation, while the corresponding tree-level

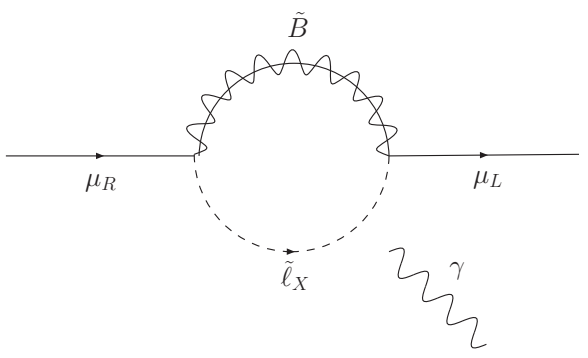

FIG. 1: Self-energy contribution $\Sigma_{22, A}^{\ell L R}$ constituting $m_{\mu}$ for $y_{\mu}=0 . \tilde{\ell}_{X}, X=1, \ldots 6$ are the charged-slepton mass eigenstates. Diagram with the attached photon contributes to $a_{\mu}$.

Yukawa couplings are zero. The radiative generation of fermion masses has several advantages compared to the general (and also the minimally flavor-violating MSSM) [11, 20, 22]:

- The otherwise approximate $[U(2)]^{5}$ flavor symmetry in the Yukawa sector becomes exact.

- It is minimally flavor-violating when only the first two generations are involved due to a natural alignment between the $A$-terms and the effective Yukawa couplings. In contrast to the naive definition of MFV (switching FCNCs mediated by gluinos and neutralinos off) the Yukawa sector is renormalisation-group invariant thanks to Symanzik's nonrenormalization theorem.

- There is no SUSY CP problem because the phases of the $A$-terms and the Yukawa couplings of the first two generations are automatically aligned. In addition, the phase of $\mu$ essentially only enters at the two-loop level (apart from a very small neutralino mixing effect) [20].

The shift in the anomalous magnetic moment depends only on the slepton and bino masses and is positive [20]. Note that this is an advantage of radiative mass generation compared to models with very large $\tan \beta$ [45, 46] , in which the discrepancy between $a_{\mu}^{\exp }$ and the theory prediction becomes larger. In the following we set $y_{\mu}=0$ and examine the phenomenological consequences for the sparticle spectrum.

As already examined in 20] (based on the analysis in [47]) vacuum stability (VS) is critical for the muon. Since the constraints from VS are nondecoupling they are equally valid for any value of $M_{\mathrm{SUSY}}$. If the muon mass is generated radiatively, the vacuum cannot be absolutely stable; only meta-stability is possible. Using the analytic trilinear term $A_{22}^{\ell}$ VS can only be satisfied for very small values of $\tan \beta \approx 1$. However, such a low value of $\tan \beta$ causes problems with the pertubativity of the top Yukawa coupling and the mass of the lightest Higgs boson. This issue can be avoided by using the nonanalytic $A$-term [48, 49, 50 $A_{22}^{\ell \prime}$ which comes with $v_{u}$ in the slepton mass matrix [50]. Note that only the combination 
$A_{22}^{\ell} v_{d}$ or $A_{22}^{\ell \prime} v_{u}$ enters in the off-diagonal element of the smuon mass matrix. One can only distinguish the different types of $A$-terms by considering Higgs-mediated processes.

In the presence of flavor-violating elements in the slepton mass matrix it is possible to generate part of the muon (and electron) mass radiatively via couplings involving $y_{\tau}$. The slepton mass eigenstates in the diagram of Fig. 1 are then linear combinations of $\tilde{\mu}_{L}, \tilde{\mu}_{R}, \tilde{\tau}_{L}$, and $\tilde{\tau}_{R}$ (and possibly also of $\tilde{e}_{L}, \tilde{e}_{R}$ ). However, by attaching a photon to the charged-slepton line one obtains the corresponding contribution to $a_{\mu}$ [60]. In addition, no chargino diagram contributes due to the absence of a tree-level Yukawa coupling. Neglecting mixing between the bino and neutral wino the magnetic moment is given as

$$
a_{\mu}=m_{\mu} \frac{\alpha_{1}}{2 \pi} M_{1} \sum_{X=1}^{6} \Re\left(Z_{L}^{2 X} Z_{L}^{5 X *}\right) m_{\tilde{\ell}_{X}}^{2} D_{0}\left(M_{1}^{2}, m_{\tilde{\ell}_{X}}^{2}, m_{\tilde{\ell}_{X}}^{2}, m_{\tilde{\ell}_{X}}^{2}\right),
$$

where $D_{0}$ is a loop function as defined in the appendix of [11]. Further the following condition must be fulfilled:

$$
m_{\mu} \stackrel{!}{=} \frac{\alpha_{1}}{4 \pi} M_{1} \sum_{X=1}^{6} Z_{L}^{2 X} Z_{L}^{5 X *} B_{0}\left(M_{1}^{2}, m_{\tilde{\ell}_{X}}^{2}\right) .
$$

We choose the diagonal elements of the slepton mass matrix to be equal such that Eq. (6) implicitly determines the off-diagonal elements. Assuming that the discrepancy in Eq. (4) can be explained within supersymmetry we can determine the allowed region in parameter space. The result for the case without flavor mixing is shown in the top left plot of Fig. 2. We see that a model with radiative generation of the muon mass predicts a lightest smuon with mass approximately between $600 \mathrm{GeV}$ and $2200 \mathrm{GeV}$ unless $M_{1}$ is heavier than $1 \mathrm{TeV}$.

With the inclusion of lepton-flavor violation it is in principle possible to weaken this bound, because e.g. $\widetilde{\mu}^{-}$ $\widetilde{\tau}$ mixing lowers the mass of one smuonlike mass eigenstate. However the effect is limited in size, because the correlation between a radiative muon mass and $a_{\mu}$ stemming from the diagrams in Fig. 1 stays intact. Moreover, the flavor-changing elements of the slepton mass matrix are tightly constrained by the radiative lepton decays $\ell_{j} \rightarrow \ell_{i} \gamma$. For a recent analysis of the bounds on the dimensionless quantities $\delta_{i j}^{\ell X Y}, X, Y=L, R$, parametrizing the off-diagonal elements of the slepton mass matrix we refer to 12]. The constraints from $\ell_{j} \rightarrow \ell_{i} \gamma$ are weakest for $\delta_{23}^{\ell R R}$ which we have kept nonzero in our analysis. The remaining elements are tightly constrained and negligible for our purpose. In the following we focus on the lighter slepton mass eigenstate. We have checked that the smuon component of this eigenstate is indeed larger than the stau component, although almost an equal mixture of $\widetilde{\mu}_{R}$ and $\widetilde{\tau}_{R}$ is possible. That is, this slepton tends to decay into muons and would be identified as a "smuon" rather than a "stau" at the LHC. We observe that the allowed area in Fig. 2 actually shrinks when $\widetilde{\mu}-\widetilde{\tau}$ mixing is included. The reason for this is that the combined effect of $\delta_{23}^{\ell R R}$ and $\delta_{22}^{\ell L R}$, which is large to account for the radiative muon mass, mimics an effective element $\delta_{23}^{\ell L R}$ which is severely constrained from $\tau \rightarrow \mu \gamma$. In the second and third plot in Fig. 2 we choose exemplarily $\delta_{23}^{\ell R R}=0.3$ and $\delta_{23}^{\ell R R}=0.5$ and include the constraint $B R(\tau \rightarrow \mu \gamma)<4.4 \times 10^{-8}$ [51] together with the condition for a radiative muon mass in Eq. (6). We recognize the two abovementioned effects: with a large $\tilde{\mu}_{L}-\tilde{\mu}_{R}-\tilde{\tau}_{R}$ mixing the allowed area moves to the left because of a lighter smuom mass eigenstate. However, $\tau \rightarrow \mu \gamma$ forbids nonvanishing $\delta_{23}^{\ell R R}$ combined with a large $\delta_{22}^{\ell L R}$ for too light smuon masses and cuts the region corresponding to lighter sparticles out. Thus, the interesting lower limit on the smuon mass in a world with soft muon Yukawa coupling stays intact in the case of flavor mixing. This is a very clean and strong prediction which gains special importance in the light of forthcoming LHC results: Since the LHC is only sensitive to light sleptons with masses $m_{\tilde{\ell}} \leq 300 \mathrm{GeV}$ at $30 \mathrm{fb}^{-1}[52,53,54]$ a detection of a smuon (in conjunction with a bino discovery or some upper bound on $M_{1}$ ) would directly disprove the hypothesis of a radiatively generated muon mass. Stated positively, a sufficiently light smuon will imply a nonzero Yukawa coupling in the MSSM superpotential. Beyond the MSSM, there is also the possibility of additional radiative contributions from sparticles with very high masses, e.g. from the messenger sector of gauge-mediated SUSY-breaking (see [45] and references therein). In such wider scenarios the question of zero or nonzero $y_{\mu}$ might profit from additional information gained from $B_{d, s} \rightarrow \mu^{+} \mu^{-}$and $B^{+} \rightarrow \mu^{+} \nu_{\mu}$ measured at the LHC and a super-B factory, respectively.

It is often stated that $a_{\mu}$ favors positive values of $\mu$ which is especially true in the large-tan $\beta$ case. However, the inclusion of the trilinear $A$-terms can compensate the effect of the $\mu$-term in the off-diagonal elements of the smuon mass matrix. This permits the possibility of negative values of $\mu$ which would otherwise be ruled out by the anomalous magnetic moment of the muon.

The discussion above applies as well to the electron and its Yukawa coupling. However, even though the anomalous magnetic moment of the electron is measured very 

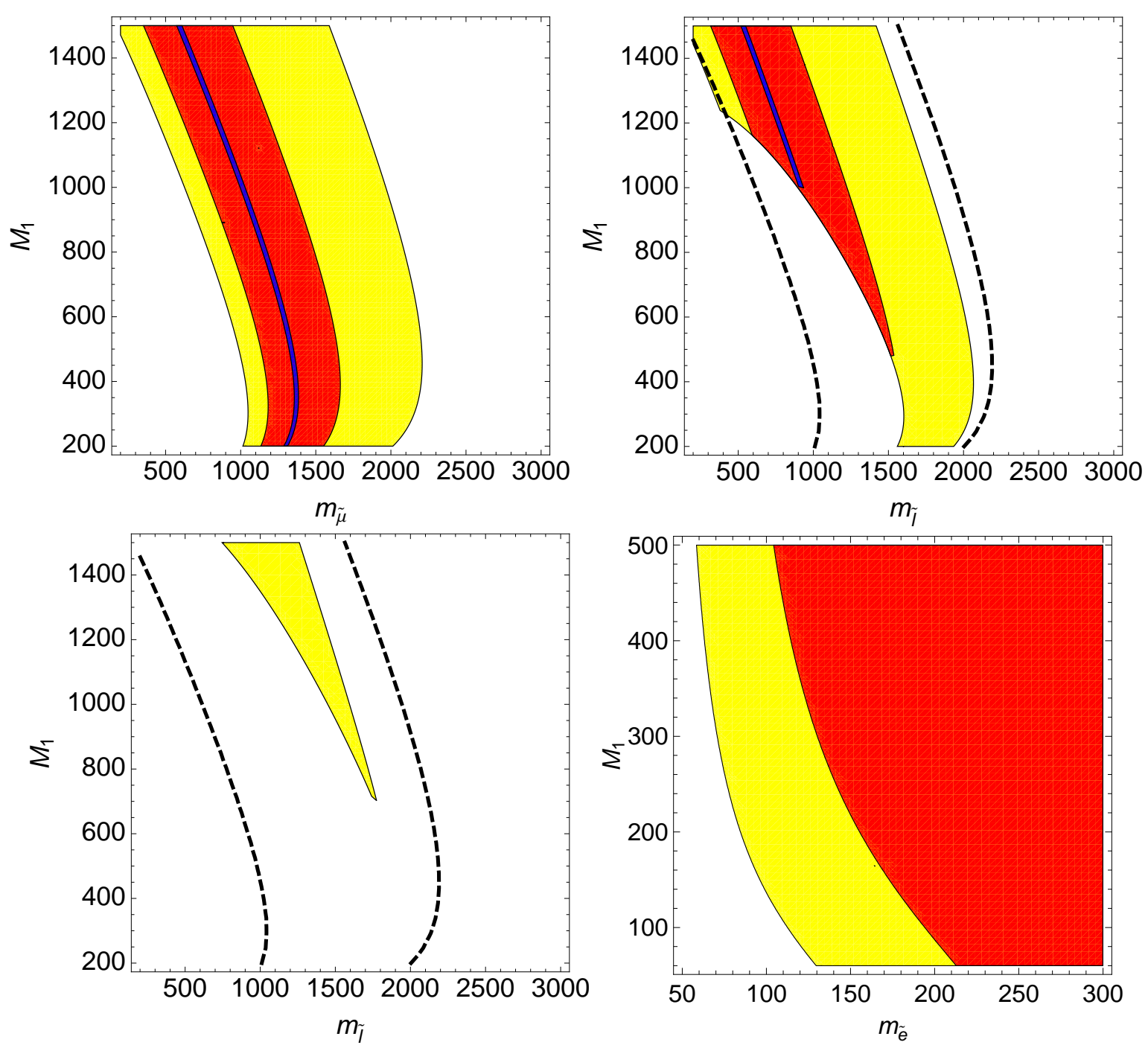

FIG. 2: Top left: Allowed region in the $M_{1}-m_{\tilde{\mu}}$ plane assuming that the muon Yukawa coupling is generated radiatively by $v_{d} A_{22}^{\ell}$ and/or $v_{u} A_{22}^{\ell \prime}$. Here $m_{\tilde{\mu}}$ is the lighter smuon mass. Yellow (lightest): $a_{\mu} \pm 2 \sigma$, red: $a_{\mu} \pm 1 \sigma$, blue (darkest): $a_{\mu}$. Top right: Allowed region in the $M_{1}-m_{\tilde{\ell}}$ plane including lepton flavor violation with $\delta_{23}^{\ell R R}=0.3$ and the constraint from $\tau \rightarrow \mu \gamma$ (black dashed for $\delta_{23}^{\ell R R}=0$ ). Down left: same with $\delta_{23}^{\ell R R}=0.5$. Down right: Allowed region in the $M_{1}-m_{\tilde{e}}$ plane assuming that the electron Yukawa coupling is generated radiatively with $v_{d} A_{11}^{\ell}$ and/or $v_{u} A_{11}^{\ell \prime}$. Yellow (lightest): $a_{e} \pm 2 \sigma$, red: $a_{e} \pm 1 \sigma$.

precisely [55], it is used to determine $\alpha$. Therefore, in order to use the anomalous magnetic moment of the electron to put bounds on new physics parameters we need an independent determination of $\alpha$ [12]. The second best way to measure the fine structure constant is from a $\mathrm{Ru}-$ bidium atom experiment [56]. Using this information we can qualitatively make the same statements as in the muon case. However, quantitatively the constraints are weaker due to the smallness of the electron mass and the uncertainty coming from the second-best measurement of $\alpha$ (see Fig. 2 down right).

\section{CONCLUSIONS}

The anomalous magnetic moment of the muon can be seen as a probe of a tree-level muon Yukawa coupling. In this paper we have performed an updated analysis of the consequences of a radiatively generated muon mass. We have found that this model is very predictive if we use the new results for the anomalous magnetic moment of the muon: The smuon mass must lie between $600 \mathrm{GeV}$ and $2200 \mathrm{GeV}$ for $M_{1}<1 \mathrm{TeV}$. The inclusion of leptonflavor violation does not significantly change the picture. This is mainly due to the fact that the same diagrams also occur for the magnetic moment. In our analysis we have included a nonvanishing $\delta_{23}^{\ell R R}$, which cannot be constrained from $\tau \rightarrow \mu \gamma$ alone. In principle, this could 
have decreased the lower bound on the smuon mass, but in conjunction with a large $\delta_{22}^{\ell L R}$ from a radiative muon mass, the constraint from $\tau \rightarrow \mu \gamma$ forbids this possibility. Consequently, the inclusion of lepton flavor violation cannot lower the bound of approximately $600 \mathrm{GeV}$ for the mass of the lightest smuonlike slepton. If this smuon is found to be lighter, the observed muon mass cannot entirely stem from the soft SUSY-breaking sector. Consequently, within the MSSM we must then have a nonzero Yukawa coupling $y_{\mu}$ in the superpotential. Therefore, we conclude that the high $p_{T}$ experiments at the LHC can shed light on the question whether $y_{\mu}$ is zero or not if they discover a smuon.
Acknowledgments. - This work is supported by BMBF Grant No.05H09VKF and by the EU Contract No. MRTN-CT-2006-035482, "FLAVIAnet". Andreas Crivellin and Jennifer Girrbach acknowledge the financial support by the State of Baden-Württemberg through Strukturiertes Promotionskolleg Elementarteilchenphysik und Astroteilchenphysik and the Studienstiftung des deutschen Volkes, respectively. A.C. is partially supported by the Swiss National Foundation. The Albert Einstein Center for Fundamental Physics is supported by the "Innovations- und Kooperationsprojekt C-13 of the Schweizerische Universitätskonferenz SUK/CRUS."
[1] T. Banks, Nucl. Phys. B303, 172 (1988).

[2] L. J. Hall, R. Rattazzi, and U. Sarid, Phys. Rev. D50, 7048 (1994), hep-ph/9306309.

[3] R. Hempfling, Phys. Rev. D49, 6168 (1994).

[4] T. Blazek, S. Raby, and S. Pokorski, Phys. Rev. D52, 4151 (1995), hep-ph/9504364.

[5] C. Hamzaoui, M. Pospelov, and M. Toharia, Phys. Rev. D59, 095005 (1999), hep-ph/9807350.

[6] G. Isidori and A. Retico, JHEP 11, 001 (2001), hep$\mathrm{ph} / 0110121$.

[7] A. J. Buras, P. H. Chankowski, J. Rosiek, and L. Slawianowska, Nucl. Phys. B659, 3 (2003), hep-ph/0210145.

[8] M. Gorbahn, S. Jager, U. Nierste, and S. Trine, arXiv:0901.2065 [hep-ph] (2009), 0901.2065.

[9] M. Carena, D. Garcia, U. Nierste, and C. E. M. Wagner, Nucl. Phys. B577, 88 (2000), hep-ph/9912516.

[10] L. Hofer, U. Nierste, and D. Scherer, JHEP 10, 081 (2009), 0907.5408.

[11] A. Crivellin and U. Nierste, Phys. Rev. D79, 035018 (2009), 0810.1613.

[12] J. Girrbach, S. Mertens, U. Nierste, and S. Wiesenfeldt, JHEP 05, 026 (2010), 0910.2663.

[13] S. Weinberg, Phys. Rev. Lett. 29, 388 (1972).

[14] A. B. Lahanas and D. Wyler, Phys. Lett. B122, 258 (1983).

[15] W. Buchmuller and D. Wyler, Phys. Lett. B121, 321 (1983).

[16] D. V. Nanopoulos and M. Srednicki, Phys. Lett. B124, 37 (1983).

[17] A. Masiero, D. V. Nanopoulos, and K. Tamvakis, Phys. Lett. B126, 337 (1983).

[18] K. S. Babu and E. Ma, Mod. Phys. Lett. A4, 1975 (1989).

[19] K. S. Babu, B. Dutta, and R. N. Mohapatra, Phys. Rev. D60, 095004 (1999), hep-ph/9812421.

[20] F. Borzumati, G. R. Farrar, N. Polonsky, and S. D. Thomas, Nucl. Phys. B555, 53 (1999), hep-ph/9902443.

[21] J. Ferrandis and N. Haba, Phys. Rev. D70, 055003 (2004), hep-ph/0404077.

[22] A. Crivellin (2009), 0905.3130.

[23] A. Crivellin and J. Girrbach, Phys. Rev. D81, 076001 (2010), 1002.0227.

[24] G. W. Bennett et al. (Muon G-2), Phys. Rev. D73, 072003 (2006), hep-ex/0602035.

[25] F. Jegerlehner and A. Nyffeler, Phys. Rept. 477, 1 (2009), 0902.3360.
[26] M. Davier, A. Hoecker, B. Malaescu, C. Z. Yuan, and Z. Zhang, Eur. Phys. J. C66, 1 (2010), 0908.4300.

[27] T. Teubner, K. Hagiwara, R. Liao, A. D. Martin, and D. Nomura (2010), 1001.5401.

[28] M. Passera, W. J. Marciano, and A. Sirlin (2010), 1001.4528 .

[29] J. Prades, Acta Phys.Polon.Supp. 3, 75 (2010), 0909.2546 .

[30] M. Davier, A. Hoecker, B. Malaescu, and Z. Zhang, Eur.Phys.J. C71, 1515 (2011), 1010.4180.

[31] R. M. Carey et al. (New $(g-2)$ Collaboration), FermilabProposal-0989 (2009), http://lss.fnal.gov/archive/testproposal/0000/fermilab-proposal-0989.pdf.

[32] S. Ferrara and E. Remiddi, Phys. Lett. B53, 347 (1974).

[33] J. A. Grifols and A. Mendez, Phys. Rev. D26, 1809 (1982).

[34] J. R. Ellis, J. S. Hagelin, and D. V. Nanopoulos, Phys. Lett. B116, 283 (1982).

[35] R. Barbieri and L. Maiani, Phys. Lett. B117, 203 (1982).

[36] D. A. Kosower, L. M. Krauss, and N. Sakai, Phys. Lett. B133, 305 (1983).

[37] T. C. Yuan, R. L. Arnowitt, A. H. Chamseddine, and P. Nath, Zeit. Phys. C26, 407 (1984).

[38] M. S. Carena, G. F. Giudice, and C. E. M. Wagner, Phys. Lett. B390, 234 (1997), hep-ph/9610233.

[39] A. Dedes, H. K. Dreiner, and U. Nierste, Phys. Rev. Lett. 87, 251804 (2001), hep-ph/0108037.

[40] D. Stockinger, J. Phys. G34, R45 (2007), hep$\mathrm{ph} / 0609168$.

[41] S. Marchetti, S. Mertens, U. Nierste, and D. Stockinger, Phys. Rev. D79, 013010 (2009), 0808.1530.

[42] G. Isidori and P. Paradisi, Phys. Lett. B639, 499 (2006), hep-ph/0605012.

[43] U. Nierste, S. Trine, and S. Westhoff, Phys.Rev. D78, 015006 (2008), 0801.4938.

[44] D. Eriksson, F. Mahmoudi, and O. Stal, JHEP 11, 035 (2008), 0808.3551.

[45] B. A. Dobrescu and P. J. Fox (2010), 1001.3147.

[46] W. Altmannshofer and D. M. Straub, JHEP 09, 078 (2010), 1004.1993.

[47] J. F. Gunion, H. E. Haber, and M. Sher, Nucl. Phys. B306, 1 (1988).

[48] H. E. Haber and J. D. Mason, Phys. Rev. D77, 115011 (2008), 0711.2890.

[49] J. Rosiek (1995), hep-ph/9511250. 
[50] D. A. Demir, G. L. Kane, and T. T. Wang, Phys. Rev. D72, 015012 (2005), hep-ph/0503290.

[51] J. Benitez (BABAR) (2010), 1006.0314.

[52] Y. M. Andreev, S. I. Bityukov, and N. V. Krasnikov, Phys. Atom. Nucl. 68, 340 (2005), hep-ph/0402229.

[53] F. del Aguila and L. Ametller, Phys. Lett. B261, 326 (1991).

[54] H. Baer, C.-h. Chen, F. Paige, and X. Tata, Phys.Rev. D53, 6241 (1996), hep-ph/9512383.

[55] T. Aoyama, M. Hayakawa, T. Kinoshita, and M. Nio, Phys. Rev. D77, 053012 (2008), 0712.2607.
[56] M. Cadoret et al., Phys. Rev. Lett. 101, 230801 (2008), 0810.3152 .

[57] I. Masina and C. A. Savoy, Nucl.Phys. B661, 365 (2003), hep-ph/0211283.

[58] J. Hisano, M. Nagai, and P. Paradisi (2008), 0812.4283.

[59] Definitions and sign conventions of SUSY parameters are as in [49].

[60] flavor-mixing in magnetic and electric dipole moments in the MSSM is studied in the literature, see e.g. 57, 58. 